Massimiliano Castellazzi*, Stefano Pizzicotti, Ilenia Lombardo, Sarah Alfiero, Andrea Morotti, Patrizia Pellegatti, Giovanna Negri, Lara Natali, Caterina Ferri, Enrico Fainardi, Tiziana Bellini and Maura Pugliatti

\title{
Sexual dimorphism in the cerebrospinal fluid total protein content
}

https://doi.org/10.1515/cclm-2020-0419

Received April 1, 2020; accepted May 18, 2020; published online June 29, 2020

\begin{abstract}
Objectives: Cerebrospinal fluid (CSF) is a clear, colorless body fluid filling the central nervous system. The determination of the CSF total protein (TP) content represents an important screening test of various pathologies. We aimed to address the effect of sex and age on CSF-TP content and the use of the current upper reference limits (URLs).

Methods: CSF-TP content was analysed in a selected population of 1,252 patients ( 648 women and 604 men; age 18-89 years) who underwent lumbar puncture as a part of the diagnostic work-up. Samples presenting (i) more than 5 white blood cells (WBC)/ $\mu \mathrm{L}$, (ii) discolorations and (iii) reduced glucose were not included.
\end{abstract}

*Corresponding author: Massimiliano Castellazzi, PhD, Department of Biomedical and Specialist Surgical Sciences, University of Ferrara, Ferrara, Italy; and Interdepartmental Research Center for the Study of Multiple Sclerosis and Inflammatory and Degenerative Diseases of the Nervous System, University of Ferrara, Ferrara, Italy, Phone: +390532 236388, E-mail: massimiliano.castellazzi@unife.it

Stefano Pizzicotti, Patrizia Pellegatti, Giovanna Negri and Lara Natali: Chemical-Clinical Analysis Laboratory, “S. Anna” University Hospital, Ferrara, Italy

Ilenia Lombardo, Sarah Alfiero and Caterina Ferri: Department of Biomedical and Specialist Surgical Sciences, University of Ferrara, Ferrara, Italy

Andrea Morotti: Stroke Unit, IRCCS Mondino Foundation, Pavia, Italy Enrico Fainardi: Department of Experimental and Clinical Biomedical Sciences, University of Florence, Florence, Italy; Interdepartmental Research Center for the Study of Multiple Sclerosis and Inflammatory and Degenerative Diseases of the Nervous System, University of Ferrara, Ferrara, Italy

Tiziana Bellini: Department of Biomedical and Specialist Surgical Sciences, University of Ferrara, Ferrara, Italy; University Center for Studies on Gender Medicine, University of Ferrara, Ferrara, Italy Maura Pugliatti: Department of Biomedical and Specialist Surgical Sciences, University of Ferrara, Ferrara, Italy; Interdepartmental Research Center for the Study of Multiple Sclerosis and Inflammatory and Degenerative Diseases of the Nervous System, University of Ferrara, Ferrara, Italy
Results: The CSF-TP content median values were significantly higher in men than in women ( $46 \mathrm{vs.} 37 \mathrm{mg} / \mathrm{dL}$ ) even after adjusting for age and different hospital inpatients. CSF-TP content positively correlated with age both in men and in women with a constant difference between sexes of $8.5 \mathrm{mg} / \mathrm{dL}$. Applying the most used URLs (mainly 45 and $50 \mathrm{mg} / \mathrm{dL}$, but also $60 \mathrm{mg} / \mathrm{dL}$ ), men received a laboratory report suggestive of altered CSF-TP content more frequently than women. The use of age- and sex-calibrated CSF-TP URLs reduced, but not eliminated, this sex-gap.

Conclusions: Using the current URLs, a condition of "elevated CSF-TP content" may be overestimated in men or, conversely, underestimated in women, regardless of the age and of the diagnosis. These results highlighted the need to apply CSF-TP URLs values normalized for both sex and age.

Keywords: age; biomarker; cerebrospinal fluid; sex; total protein content.

\section{Introduction}

Cerebrospinal fluid (CSF) is a clear, colorless body fluid filling the brain and the spinal cord. The CSF composition is very similar to a plasma ultrafiltrate with low protein concentration and few cells [1]. The diagnostic lumbar puncture, or spinal tap, is a mini-invasive procedure used to withdraw CSF from the spinal subarachnoid space [2]. With the only exception of brain biopsy, the CSF examination represents the only tool available to investigate the occurrence of (i) inflammatory, (ii) infectious, (iii) degenerative conditions, as well as (iv) the presence of CT-negative subarachnoidal haemorrhage and (v) of leptomeningeal metastases [3, 4].

CSF chemical and physical analyses are usually performed within $2 \mathrm{~h}$ from time of collection, and yield information on the fluid appearance, presence of discolorations, glucose content, circulating cell count and protein content [5].

Although the CSF/serum albumin ratio is considered the preferred marker to assess the blood-CSF-barrier (B-CSF-B) function [6], the determination of the CSF total protein (TP) content is - in routine clinical practice - an important screening test [7] for conditions such as bacterial 
meningitis, viral infections, autoimmune polyneuropathies, infectious polyneuropathies, subarachnoid hemorrhages and brain metastases [3].

An international agreement on the applicability of upper reference limits (URLs) for CSF-TP content is still lacking. A recently conducted web-based survey has disclosed that $45 \mathrm{mg} / \mathrm{dL}$ remains the most commonly used URL worldwide [8]. Higher CSF-TP content is found mainly in infants ( $<1$ year) and premature babies $[9,10]$ due to an immature B-CSF-B, and in the elderly [3, 11], yet less than $5 \%$ of the participating centers reported the use of ageadjusted reference limits for adults and/or newborns [8].

Studies on the CSF/serum albumin ratio have recently demonstrated an increase in B-CSF-B permeability to proteins in men compared to women [12, 13]. Although this may lead to a difference in CSF protein content between the two sexes, to date only a few studies have explored this aspect [14] and above mentioned sex differences have not yet been considered in the daily laboratory practice.

With this work we aimed to address the effect of sex and age on the TP content in CSF obtained from diagnostic lumbar puncture, so as to provide some scientific ground for potential reconsideration of CSF-TP reference values.

\section{Materials and methods}

\section{Study design}

The study was performed on CSF obtained for diagnostic purposes from patients who were hospitalized at the 'S. Anna' University Hospital (Azienda Ospedaliero-Universitaria S. Anna), Ferrara, northern Italy in the period 2010-2018. The study was approved by the local Committee for Medical Ethics in Research (Comitato Etico di Area Vasta Emilia Centro della Regione Emilia-Romagna, Prot. N. 770/2018/ Oss/AOUFe). Written informed consent was obtained and the data were collected in anonymized form.

Laboratory and demographic data were retrospectively collected from a population of 2,617 patients.

Exclusion criteria were the lack of demographic data (sex and/or age) and the presence of CSF abnormalities. Briefly, patients were excluded from the analysis applying these criteria: samples with (i) incomplete data ( $\mathrm{n}=92)$, (ii) CSF white blood cells $(\mathrm{WBC})>5 / \mu \mathrm{L}(\mathrm{n}=625)$, (iii) CSF discolorations ( $n=59$ ), CSF glucose $\leq 2.5 \mathrm{mmol} / \mathrm{L}$ ( $\mathrm{n}=172)$, CSF repeated samples $(n=45)$. After this first check, outliers $(n=99)$ and patients with less than 18 years $(n=273)$ were also excluded.

A population of 1,252 patients, 648 women and 604 men, was finally included in the study. Patients were analysed blindly with respect to diagnostic suspicion and definite clinical diagnosis.

\section{Sample analysis}

CSF-TP content was measured in all samples as a part of the diagnostic work-up at the Chemical-Clinical Analysis Laboratory, "S. Anna”
University Hospital (Ferrara, Italy). All the analysis was performed on the 1st-4th $\mathrm{mL}$ of CSF after lumbar puncture. CSF samples were analysed at room temperature, immediately after centrifugation and within $2 \mathrm{~h}$ from sample withdrawal with the Beckman Coulter AU640/ AU640e automated chemistry analyzer. According to the manufacturer's instructions, human serum albumin was used as a calibrator $(0.50 \mathrm{~g} / \mathrm{L})$, and intra- and inter-assays variations were $<2 \%$ and $<5 \%$ respectively. The lower detectable analyte level was estimated to be $7 \mathrm{mg} / \mathrm{dL}$, with a sample interval of 1-199 mg/dL.

\section{Statistical analysis}

Continuous variables presenting with a non-normal distribution (Kolmogorov Smirnov test) were reported as median and interquartile range (IQR). All comparisons were made with Mann-Whitney test. Categorical variables were reported as counts (percentages) and the Fisher's exact test was used for comparison. Correlations between age and CSF-TP content were investigated with Spearman test. In regression analysis F-test was used to compare the fits of linear models. Analysis of covariance (ANCOVA) was used to compare log-transformed CSF-TP content in men and women using age as covariate in the model. Furthermore, in the absence of information related to a diagnostic suspect and/or definition, the hospital units of sample provenience were grouped into intensive care, oncohaematological, infectious disorders, internal medicine, neurosurgical and neurological units, and this variable was included in the ANCOVA model as covariate. Two tailed p-values $<0.05$ were considered statistically significant. The Statistical Package for the Social Sciences (SPSS ${ }^{\circledast}$ ) version 21.0 for Windows, OSX (SPSS Inc., IBM ${ }^{\circledast}$, Somers, New York, USA) and Prism ${ }^{\circledast} 8$ (GraphPad Software Inc.) were used for the statistical analysis. Outliers were determined using the robust regression and outlier removal (ROUT) method, assuming a maximum desired false discovery rate (Q) equal to $1 \%$.

\section{Results}

\section{Patient's characteristics}

The study population women: men ratio was 1.07 . At the time of sample withdrawal, men had a higher median age than women (56 vs. 51 years; Mann-Whitney, $\mathrm{p}=0.0001$ ). The age range was the same for men and women: 18-79 years. The distribution of patients in the various age groups is shown in Table 1.

\section{Cerebrospinal fluid total protein concentrations in the study population}

The CSF-TP content median values were significantly higher in men than in women in the overall study population (46 vs. $37 \mathrm{mg} / \mathrm{dL}$ ) (Figure 1A). Stratifying the patients by age groups, the CSF-TP content was significantly higher in men than women in all age groups: $18-30$ year ( $34 \mathrm{vs.} 32 \mathrm{mg} / \mathrm{dL}$ ); $31-40$ years ( $44 \mathrm{vs} .34 \mathrm{mg} / \mathrm{dL}$ ); $41-50$ years ( $45 \mathrm{vs.} 33 \mathrm{mg} / \mathrm{dL}$ ); 51-60 years ( $51 \mathrm{vs.} 41 \mathrm{mg} / \mathrm{dL}$ ); 61-70 years (50 vs. $39 \mathrm{mg} / \mathrm{dL}$ ); over 70 years ( 46.5 vs. $40 \mathrm{mg} / \mathrm{dL}$ ) (Figure 1B). 
Table 1: Patients' age distribution.

\begin{tabular}{lrr}
\hline Age class, years & Men, $\mathbf{n}(\%)$ & Women, $\mathbf{n}(\%)$ \\
\hline $18-30$ & $59(41.5)$ & $83(58.5)$ \\
$31-40$ & $70(39.5)$ & $107(60.5)$ \\
$41-50$ & $117(48.1)$ & $126(51.9)$ \\
$51-60$ & $104(44.8)$ & $128(55.2)$ \\
$61-70$ & $132(52.6)$ & $119(47.4)$ \\
over 70 & $122(58.9)$ & $85(41.1)$ \\
Total & $604(48.2)$ & $648(51.8)$ \\
\hline
\end{tabular}

In a multivariate model, natural log-transformed (ln) CSF-TP mean content $(\mathrm{mg} / \mathrm{dL})$ was significantly higher in men than in women even after adjusting for age at the time of lumbar puncture (3.80 vs. 3.59) and different hospital units of sample provenience (3.80 vs. 3.59) (Table 2).

\section{Correlation between age, sex and cerebrospinal fluid total protein}

Age positively correlated to CSF-TP content in both women (Spearman, $\mathrm{r}=0.2086 ; \mathrm{p}<0.0001$ ) and men (Spearman, $\mathrm{r}=0.1540 ; \mathrm{p}<0.0001)$. The difference between the slopes of the two sex-specific regression lines was not significant $(\mathrm{F}=0.007339 ; \mathrm{p}=0.9317)$ while the difference between the elevations was significant $(F=76.32 ; p<0.0001)$ indicating a constant gap of $8.5 \mathrm{mg} / \mathrm{dL}$ between men and women over the age of 18 (Figure 2).

\section{Use of different reference threshold values for the cerebrospinal fluid total protein concentrations}

In our overall study population the frequency of an elevated CSF-TP content was analysed in men and women. Different threshold values were used. In particular, the following URLs were applied: $45 \mathrm{mg} / \mathrm{dL}$, i. e., the URL used in our practice [14], $50 \mathrm{mg} / \mathrm{dL}$ and, only for patients over 30 years old, $60 \mathrm{mg} / \mathrm{dL}$ [11]. As reported in Table 3, men featured CSF-TP content above the thresholds more frequently than women using the URL of $45 \mathrm{mg} / \mathrm{dL}$ (50.2 vs. $28.9 \%$ ), the URL of $50 \mathrm{mg} /$ dL (41.4 vs. $22.1 \%$ ) and the URL of $60 \mathrm{mg} / \mathrm{dL}$ (27.9 vs. $11 \%$ ).

A difference between the two sexes was also observed with the use of age- and sex-adjusted URLs as previously proposed [14]. Although the use of these thresholds reduced the percentage of positives in men $(20.9 \%)$ and women $(13 \%)$ and the gap between the two sexes was smaller, it still remained statistically significant $(p=0.0002)$ (Table 4). Interesting, these positivities were found only in three of 14 age subgroups, in particular men had CSF-TP content higher than the specific URL more frequently than women in the $41-45$ (23.4 vs. 5.7\%), 56-60 (24.1 vs.6.9\%) and $81-85$ (40 vs. 5.9\%) years subgroups.

\section{Discussion}

Our study shows that CSF-TP content is higher in men than women at all ages in a large population of adult patients undergoing lumbar puncture for diagnostic purposes. Being CSF-TP determination a widely used screening test for diverse neurological conditions [7], our findings show how abnormal CSF-TP content is more frequently reported for male than female individuals, irrespective of age.

The CSF-TP analysis is a laboratory tool commonly used in clinical practice, and a useful diagnostic support already in the earliest stages of the patient's clinical condition, by virtue of the short time needed for analysis and report from sample withdrawal [3].

An increased CSF-TP content with age has been reported in the literature [7] which could be explained by an increase B-CSF-B permeability $[6,15,16]$ and a progressive
A

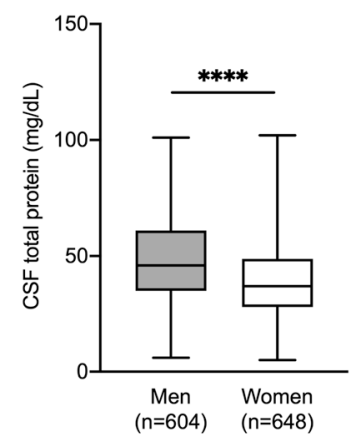

B

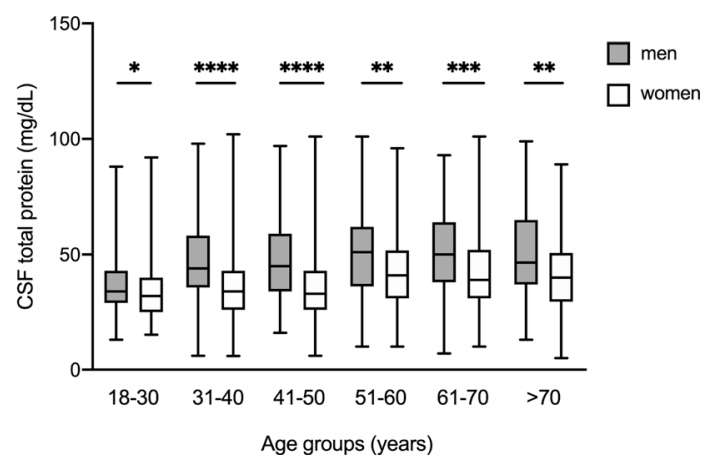

Figure 1: Cerebrospinal fluid (CSF) total protein (TP) levels in the study population analysed as a whole (A) and stratified by age (B).

Mann-Whitney U-test was used for all comparisons. CSF-TP content was higher in men than in women in the entire population $(p<0.0001)(A)$ and in all age groups: $18-30$ years $(p=0.0474) ; 31-40$ years ( $p>0.0001)$; 41-50 years ( $\mathrm{p}<0.0001) ; 51-60$ years $(p=0.0016) ; 61-70$ years $(p=0.0002) ;>70$ years $(p=0.0015)(B)$. The line within the box indicates the median. The boundaries of the box represent the 25 th-75th quartiles. The whiskers above and below the box correspond to the highest and lowest values. 
Table 2: Sex-specific mean (95\% confidence intervals, $\mathrm{Cl}$ ) of the natural log-transformed (In) cerebrospinal fluid (CSF) total protein (TP) content $(\mathrm{mg} / \mathrm{dL})$.

\begin{tabular}{|c|c|c|c|c|c|c|c|c|}
\hline Men & Women & p-Value ${ }^{a}$ & Men & Women & p-Value ${ }^{b}$ & Men & Women & p-Value \\
\hline 3.807 & 3.583 & $<0.000001$ & 3.801 & 3.589 & $<0.000001$ & 3.804 & 3.589 & $<0.000001$ \\
\hline (3.772-3.841) & (3.549-3.618) & & $(3.767-3.836)$ & $(3.555-3.622)$ & & $(3.769-3.839)$ & $(3.555-3.622)$ & \\
\hline
\end{tabular}

Men $=604$ (48.2\%); Women $=648$ (51.8\%). Natural log-transformed (In) distribution for CSF-TP content (mg/dL) and age at lumbar puncture (years). ${ }^{a}$ Student's t-test for independent variables, crude means. ${ }^{b}$ Model 1 (ANCOVA): marginal means adjusted for In-transformed age at lumbar puncture. 'Model 2 (ANCOVA): marginal means adjusted for ln-transformed age at lumbar puncture and hospital units of samples provenience. Bold p-values = statistically significant.

reduction of the CSF turnover both associated to ageing $[1,17]$. The assessment of CSF-TP content has little diagnostic value in newborns' neurological diseases due to the large variation for this parameter as a consequence of birth trauma and of the immaturity of the brain barriers $[7,10]$.

Recent studies propose age-dependent reference values for CSF-TP content $[8,11,14]$. In particular, McCudden and colleagues highlighted that the URL of $0.45 \mathrm{mg} / \mathrm{dL}$, currently the most used reference value worldwide, could produce $25 \%$ false positive patients aged over 65 years in the absence of neurological diseases [14]. In the attempt to reduce this ageing effect, some authors have proposed to use increasing URL values (e. g. $0.50 \mathrm{mg} /$ $\mathrm{dL}$ for patients below age 30 and $0.60 \mathrm{mg} / \mathrm{dL}$ for older patients), but at present these thresholds have not yet entered into clinical practice anywhere in the world $[8,11]$.

Not only does age affect the CSF composition but sex too seems to play an important role. Despite higher contents of glucose, albumin, gamma globulins and TPs were reported in men as compared to women [18], these differences were never considered in setting the URLs for these analytes.

Recently, a $6 \mathrm{mg} / \mathrm{dL}$ difference was reported for CSF-TP content between men and women [14], yet sex-adjusted URL are highly overlooked in clinical practice.
Our present work is in line with this evidence, but we highlight a larger difference between the two sexes at all age groups, of both the median value and the linear regression coefficients, and of about $8.5 / 9.0 \mathrm{mg} / \mathrm{dL}$. This observed difference from the cited work [14] appears to be partially artefactual due to the samples selection procedures. McCudden and coworkers excluded samples from patients with diseases that could increase of the CSF-TP content which however accounted only for nearly $19 \%$ of the pre-selected samples. In our work, while clinical diagnoses were not available for any of the samples, the hospital unit of provenience was known for each sample

Table 3: Frequency of increased cerebrospinal fluid (CSF) total protein (TP) concentrations using different upper reference limits (URL).

\begin{tabular}{lrrr}
\hline & Men & Women & p-Value \\
\hline Altered CSF TP, $\mathrm{n}(\%)$ & & & \\
URL $45 \mathrm{mg} / \mathrm{dL}$ & $303(50,2)$ & $187(28.9)$ & $<\mathbf{0 . 0 0 0 1}$ \\
URL $50 \mathrm{mg} / \mathrm{dL}$ & $250(41.4)$ & $143(22.1)$ & $<\mathbf{0 . 0 0 0 1}$ \\
URL $60 \mathrm{mg} / \mathrm{dL}$ & $152(27.9)$ & $62(11.0)$ & $<\mathbf{0 . 0 0 0 1}$ \\
\hline
\end{tabular}

Fisher's exact test was used for all comparisons. Bold $\mathrm{p}$-values $=$ statistically significant.

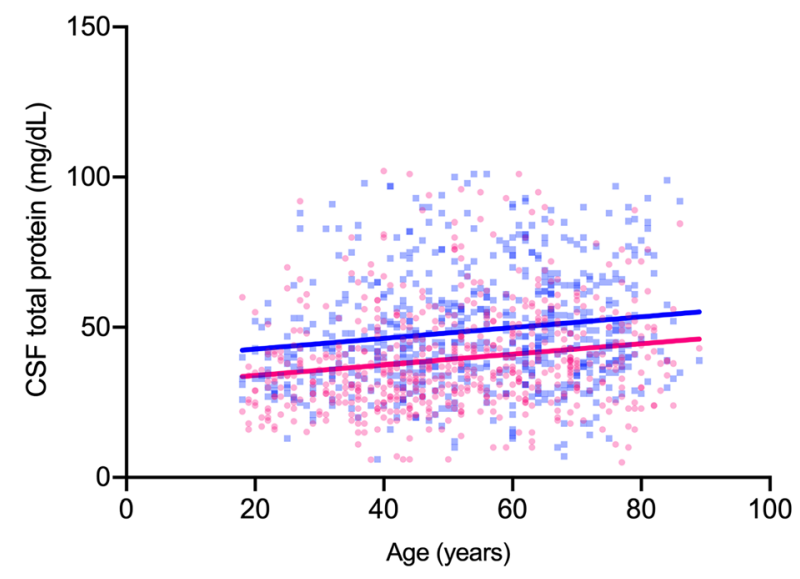

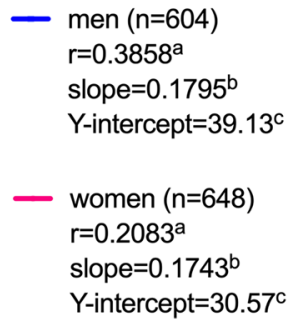

Y-intercept $=30.57^{\circ}$
Figure 2: Linear regression analysis of cerebrospinal fluid (CSF) total protein (TP) content and age in men and women. ${ }^{a}$ CSF TP and age were positively correlated both in men (Spearman, $p<0.0001$ ) and women (Spearman, $p<0.0001) .{ }^{b}$ The difference between the slopes was not significant $(F=0.007339 ; p=0.9317)$ while 'the difference between the elevations was significant $(F=76.32 ; p<0.0001)$. 
Table 4: Frequency of increased cerebrospinal fluid (CSF) total protein (TP) concentrations using upper reference limits (URL) adjusted for age according to McCudden and colleagues [14].

\begin{tabular}{|c|c|c|c|c|c|}
\hline & \multicolumn{2}{|r|}{ Men } & \multicolumn{2}{|r|}{ Women } & \multirow[t]{2}{*}{ p-Value } \\
\hline & URL, mg/dL & Positive, n (\%) & URL, mg/dL & Positive, n (\%) & \\
\hline ALL & & $126(20.9)$ & & $84(13.0)$ & 0.0002 \\
\hline \multicolumn{6}{|c|}{ Age, years } \\
\hline $18-25$ & 50 & $3(9.1)$ & 46 & $5(10.2)$ & $>0.9999$ \\
\hline $26-30$ & 54 & $4(15.4)$ & 47 & $8(23.5)$ & 0.5258 \\
\hline $31-35$ & 57 & $9(25.7)$ & 49 & $5(12.2)$ & 0.1495 \\
\hline $36-40$ & 59 & $9(25.7)$ & 51 & $15(22.7)$ & 0.8076 \\
\hline $41-45$ & 60 & $15(23.4)$ & 54 & $4(5.7)$ & 0.0053 \\
\hline $46-50$ & 61 & $12(22.6)$ & 56 & $6(10.7)$ & 0.1230 \\
\hline $51-55$ & 62 & $12(24.0)$ & 58 & $16(22.9)$ & $>0.9999$ \\
\hline $56-60$ & 63 & $13(24.1)$ & 60 & $4(6.9)$ & 0.0165 \\
\hline $61-65$ & 64 & $21(29.6)$ & 61 & $9(15.5)$ & 0.0928 \\
\hline $66-70$ & 66 & 7 (11.5) & 63 & $4(6.6)$ & 0.5292 \\
\hline $71-75$ & 68 & $5(8.3)$ & 64 & $2(8.0)$ & $>0.9999$ \\
\hline $76-80$ & 70 & $9(20.5)$ & 67 & $4(9.8)$ & 0.2317 \\
\hline $81-85$ & 72 & $6(40.0)$ & 68 & $1(5.9)$ & 0.0330 \\
\hline$>85$ & 76 & $1(33.3)$ & 72 & $1(50.0)$ & $>0.9999$ \\
\hline
\end{tabular}

Fisher's exact test was used for all comparisons. Bold $\mathrm{p}$-values = statistically significant.

and the analysis was adjusted for this covariate which had no impact on the effect of sex over the CSF-TP content.

Moreover and to the best of our knowledge, we show for the first time a nearly fixed sex difference of CSF-TP content over the age of 18, and with parallel increase with age.

Proteins cross through the B-CSF-B by receptor-mediated transcytosis/endocytosis or by fluid phase absorption $[19,20]$. Although no sex-related differences have been reported in the literature for these mechanisms, we now know that the B-CSF-B permeability to proteins is greater in men than in women, regardless of age and disease [12, 13], a mechanism which is likely ascribed to female hormones [21, 22] modulating a different expression of enzymes involved in the remodeling and turnover of the B-CSF-B [23, 24].

Confirming the effect of sex on the CSF-TP content, our study reiterates the need to re-define the application of the URLs for this analyte, which should be normalized for age and sex, in order to overcome the overestimation of CSF-TP content in men or, conversely, the underestimation in women, regardless of the age. Interestingly, in our study population, the use of URLs normalized by age and gender according to McCudden and co-workers [14] reduces this sex gap only partially.

Our study has some limitations. Due to the invasive nature of the lumbar puncture, which is performed for diagnostic or therapeutic purposes, our analyses did not include a population of healthy controls. To overcome such limitation, several studies have attempted to derive upper reference values from patient samples $[11,25]$. As in our study, the effect of peripheral blood contamination was minimized by removing the CSF samples with discolorations or increased WBC. Moreover, the lack of clinical diagnoses did not allow us to exclude patients with clinical conditions associated with a high CSF-TP content and this could account for the slightly higher CSF-TP levels as compared to studies conducted on "normal" subjects [14].

In conclusion, our study confirms a sexual dimorphism in the CSF-TP with a greater CSF-TP content more frequently assessed in men than in women, and that its values exceed the most commonly URLs references ( $45 \mathrm{mg} /$ $\mathrm{dL}$ and $50 \mathrm{mg} / \mathrm{dL}$ ).

In the era of precision medicine, better normalized CSF-TP reference values will have relevant implications on practice, adding further value to the CSF analysis, a preciously informative and quickly accessible 'window' to the CNS.

We encourage the use of sex- and age-normalized URLs in the daily CSF-TP analysis, as well as the search of the best sex- and age-specific threshold values by implementing a network of expertise and a larger study population, possibly including healthy CSF donors.

Acknowledgments: We acknowledge Fondazione Banca Italia for having contributed to creating an academic network for discussion on the topic, by supporting an event entitled Cerebrospinal fluid in neuroinflammation and neurodegeneration: updates on diagnostic and prognostic opportunities (Ferrara, Italy, November 22 2019). 
Research funding: None declared.

Author contributions: MC participated in study concept and design and drafted the manuscript. AM participated in analysis and interpretation of data. SP, IL, SA, PP, GN and LN participated in acquisition and analysis of data. MC, EF, TB and MP participated in interpretation of data and critically revised the manuscript. All authors have accepted responsibility for the entire content of this manuscript and approved its submission.

Competing interests: Authors state no conflict of interest. Informed consent: Informed consent was obtained from all individuals included in this study.

Ethical approval: The study was approved by the local Committee for Medical Ethics in Research (Comitato Etico di Area Vasta Emilia Centro della Regione EmiliaRomagna, Prot. N. 770/2018/Oss/AOUFe).

Availability of data and materials: The datasets used and analysed during the current study are available from the corresponding author on reasonable request.

\section{References}

1. Hühmer AF, Biringer RG, Amato H, Fonteh AN, Harrington MG. Protein analysis in human cerebrospinal fluid: Physiological aspects, current progress and future challenges. Dis Markers 2006;22:3-26.

2. Roos KL. Lumbar puncture. Semin Neurol 2003;23:105-14.

3. Deisenhammer F, Bartos A, Egg R, Gilhus NE, Giovannoni G, Rauer $\mathrm{S}$, et al. Guidelines on routine cerebrospinal fluid analysis. Report from an EFNS task force. Eur J Neurol 2006;13:913-22.

4. Gastaldi M, Zardini E, Leante R, Ruggieri M, Costa G, Cocco E, et al. Cerebrospinal fluid analysis and the determination of oligoclonal bands. Neurol Sci 2017;38:217-224.

5. Smith SV, Forman DT. Laboratory analysis of cerebrospinal fluid. Clin Lab Sci 1994;7:32-8.

6. Andersson M, Alvarez-Cermeno J, Bernardi G, Cogato I, Fredman P, Frederiksen J, et al. Cerebrospinal fluid in the diagnosis of multiple sclerosis: a consensus report. J Neurol Neurosurg Psychiatry 1994; 57:897-902.

7. Thompson EJ, Keir G. Laboratory investigation of cerebrospinal fluid proteins. Ann Clin Biochem 1990;27:425-35.

8. Bourque PR, Breiner A, Moher D, Brooks J, Hegen H, Deisenhammer F, et al. Adult CSF total protein: Higher upper reference limits should be considered worldwide. A web-based survey. J Neurol Sci 2019;396:48-51.

9. Srinivasan L, Shah SS, Padula MA, Abbasi S, McGowan KL, Harris MC. Cerebrospinal fluid reference ranges in term and preterm infants in the neonatal intensive care unit. J Pediatr 2012;161:729-34.

10. Kahlmann V, Roodbol J, van Leeuwen N, Ramakers CRB, van Pelt D, Neuteboom RF, et al. Validated age-specific reference values for CSF total protein levels in children. Eur J Paediatr Neurol 2017;21:654-60.
11. Hegen H, Auer M, Zeileis A, Deisenhammer F. Upper reference limits for cerebrospinal fluid total protein and albumin quotient based on a large cohort of control patients: implications for increased clinical specificity. Clin Chem Lab Med 2016;54: 285-92.

12. Parrado-Fernandez C, Blennow K, Hansson M, Leoni V, CedazoMinguez A, Bjorkhem I. Evidence for sex difference in the CSF/ plasma albumin ratio in 20000 patients and 335 healthy volunteers. J Cell Mol Med 2018;22:5151-4.

13. Castellazzi M, Morotti A, Tamborino C, Alessi F, Pilotto S, Baldi E, et al. Increased age and male sex are independently associated with higher frequency of blood-cerebrospinal fluid barrier dysfunction using the albumin quotient. Fluids Barriers CNS 2020;17:14.

14. McCudden CR, Brooks J, Figurado P, Bourque PR. Cerebrospinal fluid total protein reference intervals derived from 20 years of patient data. Clin Chem 2017;63:1856-65.

15. Link H, Tibbling G. Principles of albumin and IgG analyses in neurological disorders. II. Relation of the concentration of the proteins in serum and cerebrospinal fluid. Scand J Clin Lab Invest 1977;37:391-6.

16. Reiber H. Flow rate of cerebrospinal fluid (CSF)-a concept common to normal blood-CSF barrier function and to dysfunction in neurological diseases. J Neurol Sci 1994;122:189-203.

17. Chen RL, Chen CP, Preston JE. Elevation of CSF albumin in old sheep: relations to CSF turnover and albumin extraction at bloodCSF barrier. J Neurochem 2010;113:1230-9.

18. Breebaart K, Becker $\mathrm{H}$, Jongebloed FA. Investigation of reference values of components of cerebrospinal fluid. J Clin Chem Clin Biochem 1978;16:561-5.

19. Schubert W, Frank PG, Razani B, Park DS, Chow CW, Lisanti MP. Caveolae-deficient endothelial cells show defects in the uptake and transport of albumin in vivo. J Biol Chem 2001;276: 48619-22.

20. Tiruppathi C, Naqvi T, Wu Y, Vogel SM, Minshall RD, Malik AB. Albumin mediates the transcytosis of myeloperoxidase by means of caveolae in endothelial cells. Proc Natl Acad Sci U S A 2004; 101:7699-704.

21. Na W, Lee JY, Kim WS, Yune TY, Ju BG. 17beta-estradiol ameliorates tight junction disruption via repression of MMP transcription. Mol Endocrinol 2015;29:1347-61.

22. Yin H, Wan Q, Tian Y, Zhao B, Deng Y. Female hormone 17betaestradiol downregulated MMP-2 expression and upregulated A1PI expression in human corneal stromal cells. Cell Biochem Biophys 2018;76:265-71.

23. Gu C, Wang F, Hou Z, Lv B, Wang Y, Cong X, et al. Sex-related differences in serum matrix metalloproteinase-9 screening noncalcified and mixed coronary atherosclerotic plaques in outpatients with chest pain. Heart Ves 2017;32:1424-31.

24. Castellazzi M, Ligi D, Contaldi E, Quartana D, Fonderico M, Borgatti L, et al. Multiplex matrix metalloproteinases analysis in the cerebrospinal fluid reveals potential specific patterns in multiple sclerosis patients. Front Neurol 2018;9:1080.

25. Dufour-Rainfray D, Beaufils E, Vourc'h P, Vierron E, Mereghetti L, Gendrot C, et al. Total protein level in cerebrospinal fluid is stable in elderly adults. J Am Geriatr Soc 2013;61:1819-21. 